\title{
NO NMDA receptor activity
}

\author{
David S. Bredt
}

A brain attack-until recently viewed as an untreatable stroke-is now regarded as a potentially curable medical emergency. Like its cousin the heart attack, a stroke is often caused by clotting of a blood vessel. Tissue plasminogen activator, a clot-dissolving drug given to heart attack patients, has now been shown effective in treatment of stroke ${ }^{1}$. But much of the debilitating brain damage in stroke results from "excitotoxic" cell death.

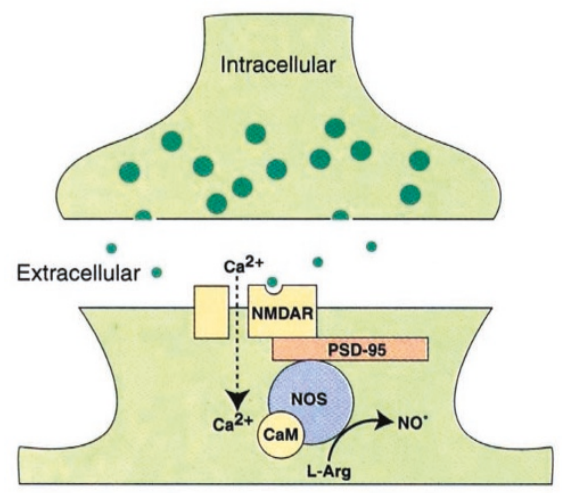

Figure 1. Coupling of NMDA receptor (NMDAR) activity to NO biosynthesis in synaptic spines. Association of NMDARs and NOS with PSD-95 protein affords efficient signal transduction at the the synapse. Abbreviations: CaM, calmodulin; Glu, glutamate; L-Arg, L-arginine.

Excitotoxicity is due to excess stimulation of neuronal NMDA ( $N$-methyl D-aspartate) type glutamate receptors and to accumulation of toxic free radicals, such as nitric oxide $(\mathrm{NO})^{2,3}$. Two articles in this issue of Nature Biotechnology describe new technologies that may be useful for the development of therapies for stroke and other free radical diseases. $\mathrm{Li}$ et al. ${ }^{4}$ select for a new class of peptide inhibitors to the NMDA receptor, and Yoshimura et $a .^{5}$ describe a method for imaging endogenous NO formation.

Physiologically, glutamate is the major excitatory neurotransmitter in brain. Acting on NMDA receptors, glutamate signaling is required for synaptic development and plasticity, processes that underlie learning and memory. At the cellular level, NMDA receptors function as glutamate-

David S. Bredt is assistant professor of physiology, department of physiology and program in biomedical sciences, University of California at San Francisco School of Medicine, 513 Parnassus Ave., San Francisco, CA 941430444 (bredt@itsa.ucsf.edu). activated calcium channels. The influx of calcium through NMDA receptors is linked to diverse cellular, metabolic, and genetic responses in the stimulated neuron.

One "second messenger" often formed in response to NMDA receptor activity is NO, a rapidly diffusible neuronal messenger ${ }^{6,7}$. Calcium, flowing through NMDA receptors, binds to calmodulin and activates NO synthase (NOS). This cascade is particularly efficient because NMDA receptors and NOS are linked together at the synapse by a common molecular bridge (see Fig. 1), the postsynaptic density-95 (PSD-95) protein ${ }^{8}$. NO influences many physiological processes mediated by NMDA receptors, including some forms of synaptic plasticity?.

This robust NO response to NMDA receptor engagement becomes the brain's enemy in certain disease states. In stroke, the lack of oxygen and other factors alters neuronal metabolism and causes excess release of glutamate. Blocking the actions of glutamate at NMDA receptors or inhibiting downstream targets, such as NOS, represent popular therapeutic strategies. Indeed, antagonists of NMDA receptors function as effective neuroprotective agents. Mice deficient in NOS are similarly resistant to brain damage in stroke models ${ }^{10}$.

Unfortunately, translating these ideas into therapeutic drugs has been difficult. Presently available NOS inhibitors limit blood flow to the brain and can actually worsen damage in stroke. Newer, more selective inhibitors of neuronal-type NOS show promise in preventing brain injury without altering blood pressure. On the other hand, potent NMDA receptor antagonists are similar to phencyclidine (PCP) or "angel-dust" and can themselves cause temporary psychosis or even permanent neuronal injury ${ }^{11}$.

To identify new classes of NMDA receptor antagonists, Li et al. use a powerful peptide selection technique. In their approach, they constructed a complex library of billions of distinct bacteriophage that each displayed a random eight amino-acid sequence on the surface of their phage coat protein. This library was screened for phage that displayed peptides that would cause the phage to bind tightly to the extracellular domain of the type 1 NMDA receptor. Those phage that associated with the NMDA receptor were selected, amplified and the screening was repeated. Interacting phage from this first screen were then randomly mutagenized and subjected to additional rounds of selection-a process referred to as molecular evolution.
This strategy eventually yielded a 16 amino-acid peptide, Mag-1.5, that bound to the NMDA receptor and functionally blocked its calcium channel activity. Previous pharmacological studies have pointed to at least six sites on the NMDA receptor that can be modulated by external compounds. Mag 1.5 appears not to interact with any of these previously identified sites.

Identification of a novel binding site on the extracellular domain of the NMDA receptor may pave the way for the design of new classes of receptor antagonists. Furthermore, this work demonstrates the utility of bacteriophage display libraries for selection of modulators of neurotransmitter-gated ion channels.

To prevent brain injury, NMDA receptor blockers should interfere with the burst of NO that accompanies excess glutamate release. But assessing NO formation is a formidable task. NO is an evanescent free radical that rapidly decomposes upon contact with cellular constituents. The new work by Yoshimura et al. demonstrates the utility of electron paramagnetic resonance (EPR) for imaging NO for the first time in an intact animal-in this case, the mouse liver.

Much like magnetic resonance imaging (MRI), which is used for high-quality brain scans, EPR allows non-invasive investigation of organs and tissues. To visualize endogenous NO by EPR, the authors first treat mice with a free-radical trapping agent that catches the short-lived NO and generates an EPR signal. The utility of this technique is limited by the NO-trapping reagent, which itself must be innocuous and needs to have access to the tissue being studied. Also, because free radicals are delocalized by trapping reagents, the resolution of EPR can never match that of MRI. Despite these limitations, EPR provides the first window for observing $\mathrm{NO}$ in vivo and may be useful in monitoring NO production in disorders, such as stroke.

1. The National Institute of Neurological Disorders and Stroke rtPA Stroke Study Group. 1995. N. Engl. J. Med. 333:1581-1587.

2. Choi, D.W. et al. 1990. Annu. Rev. Neurosci. 13:171-182.

3. Dawson, T.M.et al. 1992. Ann. Neurol. 32:297-231.

4. Li, M. et al. 1996. Nat. Biotechnol. 14:986-991.

5. Yoshimura, T. et al. 1996. Nat. Biotechnol. 14:992-994.

6. Garthwaite, J. et al. 1995. Annu Rev. Physiol. 57:683-706.

7. Bredt, D.S. et al. 1994. Annu. Rev. Biochem. 63:175-195.

8. Brenman, J.E. et al. 1996. Cell 84:757-767.

9. Schuman, E.M. et al. 1994. Annu Rev. Neurosci. 17:153-183.

10. Huang, Z. et al. 1994. Science 265:1883-1885. 11. Olney, J.W. et al. 1989. Science 244:1360-1362. 